\title{
Medición del trabajo de una línea de producción de yogurt - empresa La Hacienda Productos Alimenticios
}

Work measurement of a yogurt line production - La Hacienda Entreprise

GLORIA ELIZABETH GRIMALDO LEÓN

Ingeniera de Producción Industrial Mg. Administración y Dirección de Empresas Grupo de Investigación LOGyCA Universidad de Boyacá, Colombia gegrimaldo@uniboyaca.edu.co

\section{DENIS CAROLINA MORENO CASTILLO}

Ingeniera Industrial Grupo de Investigación LOGyCA Universidad de Boyacá, Colombia denmoreno@uniboyaca.edu.co

\section{MARÍA CAMILA SALAMANCA MOLANO}

Ingeniera Industrial Grupo de Investigación LOGyCA Universidad de Boyacá, Colombia marsalamanca@uniboyaca.edu.co

Recibido: 26/06/2014
Aceptado: 17/02/2015 


\section{RESUMEN}

El presente artículo expone los resultados de una investigación descriptiva tipo estudio de caso, realizada en la línea de producción de yogurt de una empresa de lácteos. Debido a que, la empresa no ha adelantado estudios similares, esta desconoce su capacidad real de producción, viéndose enfrentada a diversas situaciones como la incertidumbre del mercado que puede satisfacer, la cantidad de materia prima que debe adquirir, y desde luego, el número de trabajadores a contratar. En razón a ello, se decidió aplicar un estudio de tiempos, que siguiendo la metodología planteada por la Organización Internacional del Trabajo OIT, permitió establecer que el tiempo estándar para procesar 550 litros de leche es de 601,32 minutos; asimismo, se identificó que el cuello de botella del sistema corresponde a la estación de Preparación del yogurt que con un tiempo estándar de 391,19 minutos consume la mayor parte del tiempo de ciclo. De esta manera, se estableció que el índice de procesamiento o capacidad real de producción del proceso, es de 468 bolsas de yogurt/hora. Finalmente, se revisó el indicador de eficiencia de la línea y se plantearon posibles opciones de mejora.

Palabras clave: Estudio de tiempos, mejoramiento de procesos, tiempo estándar, sector lácteo.

\section{ABSTRACT}

This paper shows the results of a descriptive research, type case study, carried out in a production line of a yogurt enterprise. Due to the company has not carried out similar studies, it unknown its actual production capacity, being faced with different situations as the uncertainty of the market that can be satisfied, the amount of raw material that must be purchased, and the number of workers to hire. Because of this, it was decided to apply a times study, which according to the methodology proposed by the Work International Organization, helped to establish that the standard processing time to process 550 liters of milk is 601,32 minutes; also it was identified that the bottleneck of the system corresponds to the station of preparation of yogurt, that consumes most of the cycle time with a standard time of 391,19 minutes. In this way, it was established that the rate of processing or actual production capacity of the process, is of 468 bags of yogurt per hour. Finally, line efficiency indicator was reviewed and possible options for improvement were proposed.

Keywords: times study, process improvement, standard time, food industry. 


\section{INTRODUCCIÓN}

A pesar de que la economía colombiana creció en un 4,3\% en 2013, superando a países como Brasil y en general, a toda Latinoamérica, cuya cifra por este concepto fue del -2,6\% (Mouthon, 2014), la situación de la industria manufacturera en el país es preocupante; no solo por las cifras presentadas en los últimos dos años sobre crecimiento de la producción industrial, sino por las repercusiones que ello pueda tener en los demás sectores de la economía colombiana (Parada, 2014). Según resultados de la Encuesta de Opinión Industrial Conjunta (EOIC) de la ANDI (2014), durante 2010 y 2011 el crecimiento de la producción industrial - tras dos años consecutivos de presentar cifras negativas - fue en aumento, al alcanzar cifras del 4,5\% y el 5,9\%, respectivamente; mientras que, en 2012 el crecimiento solo fue del $0,7 \%$ y en 2013 cayó al $-0,2 \%$.

En contraste, las cifras presentadas por el DANE en relación con la producción real industrial del país, indican cómo la última crisis de la Industria manufacturera, después de la presentada entre 2008 y 2009, comenzó realmente en 2012. Lo anterior debido a que, la producción real del sector lácteo decreció en un $0,3 \%$ y un 1,9\% durante 2012 y 2013, respectivamente. Sin embargo, para el primer trimestre de 2014, el panorama para la industria manufacturera fue alentador, puesto que se estimó que la producción real industrial a marzo de 2014 había alcanzado el 4,4\%, frente a un -6,4\% registrado en marzo del año inmediatamente anterior, donde la clase industrial de los productos lácteos constituyó uno de los sectores más importantes de la industria colombiana, puesto que no solo es el tercer subsector con mayor crecimiento de su producción a marzo de $2014(8,2 \%)$ sino porque representa una oportunidad para ampliar su mercado internacional (ANDI, 2014).

Lo anterior se sustenta en que, aun cuando la Unión Europea es la productora más importante de leche en el mundo, las esperanzas en torno al crecimiento de la producción de dicho sector para esta década se centran especialmente en Latinoamérica (Montero, 2013).

Por su parte, la FAO (Food and Agriculture Organization of the United States) espera que América Latina aumente su producción lechera en un $20 \%$ entre 2013 y 2020, de forma que llegue a producir 93,8 millones de toneladas de leche en el 2020 (Montero, 2013). Asimismo, dicha organización estima que la producción de leche mundial incrementará en 168 Mt para tales fechas, de los cuales se espera que los países en desarrollo como Colombia, Argentina y Brasil, aporten el 74\% (OCDE/FAO, 2013). Luego, es evidente que la industria manufacturera colombiana y puntualmente la clase de los lácteos, requieren incrementar sus niveles de productividad, para así hacerse más competitivos en el mercado internacional. 
Entretanto, al cierre de 2013, Boyacá fue el cuarto departamento más productor de leche después de Antioquia, Córdoba y Casanare, aportando el 7,25\% del total de la producción nacional, y cuyo 21,38\% de la producción tuvo como destino la industria, la cual consumió el 44,92\% de la producción lechera nacional de 2013 (DANE, 2013). Sin embargo, los productos lácteos correspondieron a no más del 0,7\% de la producción industrial de Boyacá en 2013 (MinCIT, 2013).

La problemática es evidente y obedece fundamentalmente, según los industriales, a la competencia y al bajo nivel de productividad de la industria colombiana; razón por la cual al $58 \%$ de dicha población les atrae invertir en la modernización de la tecnología con el ánimo de mejorar los niveles de productividad de sus procesos, e igualmente a un 33,9\% le interesa tanto ampliar su planta de producción como implementar proyectos de innovación (ANDI, 2014). En tanto que, para Quintero (2011) la problemática de la industria nacional y específicamente del subsector de los productos lácteos, se cierne en torno a la baja productividad y los altos costos de producción del eslabón primario de la cadena láctea, la calidad de la leche, entre otros.

Atendiendo a la necesidad de la industria manufacturera del país y al potencial del sector de los lácteos, se decidió aplicar un estudio de tiempos en la empresa La Hacienda, la cual es una empresa boyacense dedicada desde hace 18 años a la fabricación de productos lácteos como yogurt, quesos y arequipe. Dada la falta de investigaciones, dicha empresa se ve enfrentada a dificultades como el desconocimiento de su capacidad de producción y en consecuencia, a la incertidumbre sobre qué porcentaje del mercado puede satisfacer.

Así, el objetivo de la presente investigación fue establecer el tiempo estándar y la capacidad real de producción del proceso de fabricación del yogurt (producto pionero de la empresa), mediante un estudio de tiempos; el cual la OIT (1996) define así:

El estudio del trabajo actúa como el bisturí del cirujano, exponiendo a la vista de todos las actividades y el funcionamiento, malo o bueno, de una empresa... Consiste entonces en examinar de qué manera se está realizando una actividad, simplificar o modificar el método operativo para reducir el trabajo innecesario o excesivo, o el uso antieconómico de recursos, y fijar el tiempo normal para la realización de la actividad (p. 18).

Igualmente, La Organización Internacional de Trabajo (OIT) (2005) se refiere al estudio del trabajo como un conjunto de técnicas, que se emplean para examinar el trabajo humano bajo distintos contextos para la investigación de todos los factores que influyen en la eficiencia y economía del proceso con el fin de proponer mejoras. Por tanto, se puede inferir que el objetivo principal del estudio del trabajo es el incremento de la productividad en la organización en que se aplique y por consiguiente, de la eficiencia y la eficacia, ya que "la productividad implica eficiencia y eficacia en el desempeño individual y organizacional... siendo la eficacia el cumplimiento de objetivos y la eficiencia el logro 
de las metas con la menor cantidad de recursos" (Koonts \& Weihrich, 1999). Al respecto, Niebel \& Freivalds (2004) respaldan lo anterior, afirmando que "la única posibilidad para que una empresa o negocio crezca y aumente su rentabilidad es aumentar la productividad" (p. 9).

El estudio del trabajo se compone básicamente de los estudios de tiempos y métodos, que se emplean para examinar el trabajo humano bajo distintos contextos para la investigación de todos los factores que influyen en la eficiencia y economía del proceso con el fin de proponer mejoras (OIT, 2005, p. 273). Asimismo, el estudio de tiempos es indispensable para la empresa en las actividades de planeación de la producción, establecimiento de tiempos de entrega y determinación de costos de fabricación, ya que es a partir de los tiempos de operación que ello puede calcularse (Alonso, 1998). En consecuencia, el estudio del trabajo es una herramienta fundamental a la hora de reducir los costos operacionales, eliminar tiempos improductivos y mejorar la calidad del producto (Meyers, 2000).

El presente artículo se estructura de la siguiente manera: en la sección número dos (2) se describirá: el tipo de investigación desarrollada, la metodología seleccionada para llevar a cabo el estudio de tiempos y los resultados esperados en cada una de las etapas correspondientes; en la sección tres (3), se presentarán los resultados obtenidos, analizando como contribuyen al cumplimiento del objetivo de la investigación. Finalmente, en la sección número cuatro (4), se mostrarán las conclusiones a las que se llegó con la ejecución de la investigación.

\section{METODOLOGÍA}

De acuerdo con Sampieri, Collado \& Baptista (2010), la investigación desarrollada, corresponde a una investigación aplicada tipo estudio de caso, con un nivel de alcance descriptivo. En cuanto a la estructura metodológica adoptada, se seleccionó la metodología establecida por la OIT en el tema de estudio del trabajo, puesto que, es la máxima autoridad a nivel internacional en lo relacionado con la promoción de los derechos laborales.

La metodología de la OIT (2005), plantea que la aplicación de un estudio de tiempos es un proceso que consta de ocho etapas, como se muestra en la figura 1, que comienza con la consecución de la información necesaria y termina con el cálculo del tiempo tipo o tiempo estándar de la tarea en estudio. 


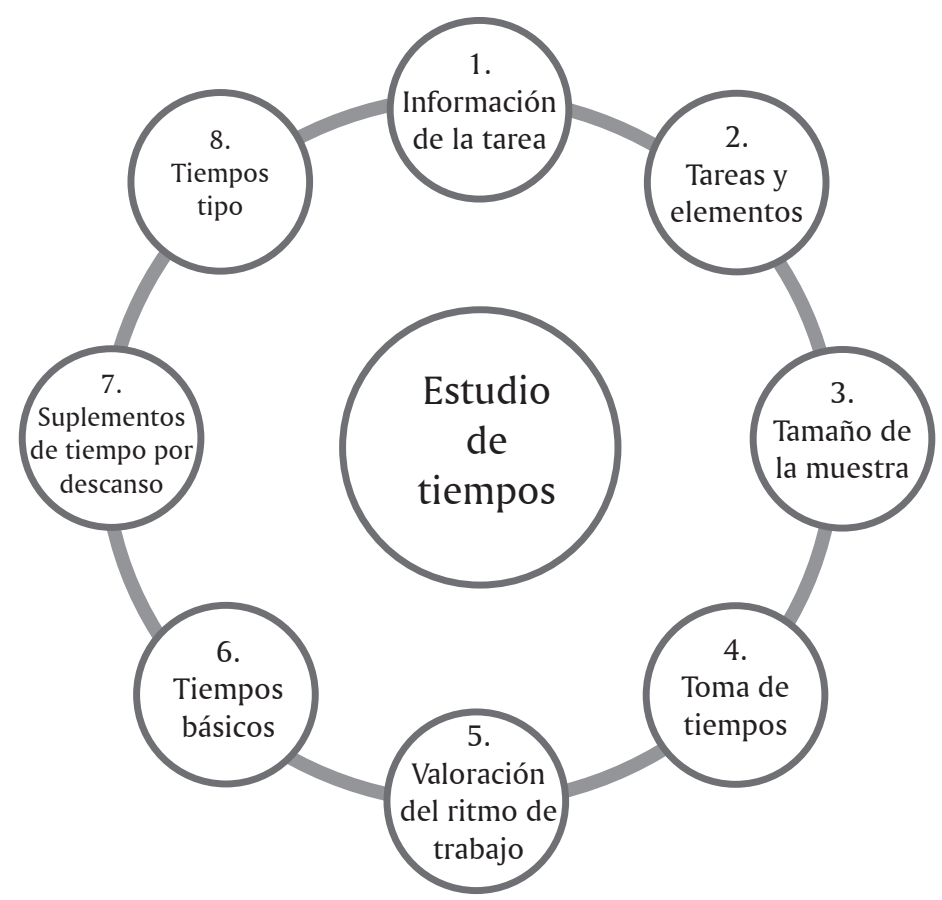

Figura 1. Metodología de la OIT para aplicar un estudio de tiempos.

Fuente: OIT, 2005.

1. Etapa 1. Información de las tareas. Mediante observación directa y entrevistas con el personal, se identificaron las tareas llevadas a cabo dentro del proceso del yogurt; así como también, los diferentes recursos que en ello intervienen. Tal información, se consolidó en un diagrama de flujo y un diagrama de recorrido del proceso, figuras 2 y 3 respectivamente.

2. Etapa 2. Tareas y elementos. Se identificaron las actividades que se ejecutaban en cada una de las tareas, es decir, sus elementos, delimitando así el inicio y fin de las posteriores tomas de tiempos.

3. Etapa 3. Tamaño de la muestra. Con tres tomas de tiempos preliminares, se estableció un valor de referencia acerca del tiempo que tardaban cada una de las tareas. A partir de ello, se aplicó la ecuación (1), que con un nivel de confianza del 95\%, arrojó tamaños de muestra de entre 8 y 17 observaciones para las diferentes tareas del proceso. 


$$
n=\left(\frac{40 \sqrt{n^{\prime} \sum x^{2}-\left(\sum x\right)^{2}}}{\sum x}\right)^{2}
$$

Ecuación 1. Tamaño de la muestra. Fuente: OIT (2005).

$n=$ Tamaño de la muestra

$n^{\prime}=$ número de observaciones del estudio preliminar

$\Sigma=$ suma de valores

$x=$ valor de las observaciones

4. Etapa 4. Observaciones de tiempo. Manejando un cronometraje de tipo acumulativo y empleando el formato general de estudio de tiempos, suministrado por la OIT (ver tabla 1), se hicieron las respectivas observaciones de tiempo, estableciéndose así, los tiempos observados de cada uno de los elementos de las tareas, componentes del proceso.

\begin{tabular}{lllll}
\hline \hline DESCRIPCIÓN DEL ELEMENTO & V. & C. & T.R. & T.B. \\
\hline \hline
\end{tabular}

Tabla 1. Formato general de estudio de tiempos. Fuente:

OIT (2005).

5. Etapa 5. Valoración del ritmo de trabajo. Simultáneo a la toma de tiempos, en el formato general de estudio de tiempos, se asignó a cada elemento de tarea (en cada observación), la valoración del ritmo con que el operario desempeñaba su trabajo. Si no había intervención de la mano de obra, no se realizaba tal asignación.

Aunque la valoración se hizo a juicio del investigador, se manejó como referencia la escala de la norma británica, que califica la velocidad de un operario con un porcentaje de entre el $0 \% \mathrm{y}$ el $150 \%$, siendo el $100 \%$ la valoración atribuida al desempeño tipo. Las diferentes asignaciones hechas por elemento, se promediaron para obtener el factor de valoración promedio, con el que luego se calcularon los tiempos básicos (OIT, 2005).

6. Etapa 6. Tiempo básico o normal. Empleando la ecuación (2), se aplicó al tiempo observado promedio de los elementos de cada tarea, el factor de valoración correspondiente. Como instrumento de recolección de la información, se utilizó el formato de hoja de estudio de tiempos, suministrado por la OIT (ver tabla 2). 


$$
\text { Tiempo básico }=\frac{\text { Tiempo observado } * \text { Valor atribuido }}{\text { Valor tipo }}
$$

Ecuación 2. Cálculo de tiempos básicos. Fuente: OIT (2005).

El valor atribuido corresponde al factor de valoración a asignar, y el valor tipo, es la valoración para un desempeño tipo, la cual siempre será del 100\% (OIT, 2005).

\begin{tabular}{llllllllllllllll}
\hline \hline \multirow{2}{*}{ EL. NÚM. } & DESCRIPCIÓN & \multicolumn{1}{c}{ TIEMPO OBSERVADO } & \multicolumn{1}{c}{ TOTAL } & PROM. & \multirow{2}{*}{ TB } \\
& DEL ELEMENTO & 1 & 2 & 3 & 4 & 5 & 6 & 7 & 8 & 9 & 10 & T.O. & T.O. & & \\
\hline \hline
\end{tabular}

Tabla 2. Formato hoja de estudio de tiempos.

Fuente: OIT (2005).

7. Etapa 7. Suplementos de tiempo por descanso. Para determinar el porcentaje de tiempo a conceder por suplementos fijos (necesidades personales y fatiga), durante el estudio de tiempos se registraron en los formatos mencionados, las condiciones de trabajo en que los operarios desempeñaban sus funciones, puntualmente en cada tarea. Por concepto de fatiga, se asignó entre un 5 y 7 por ciento; en tanto que, por descanso se atribuyó un factor constante del $4 \%$, recomendado por la OIT (2005).

Igualmente, mediante entrevistas con el personal y los directivos, se pudo establecer que solo en la estación de empaque, se manejaba un margen de tiempo de 10 minutos por contingencias (suplementos variables).

8. Etapa 8. Tiempo tipo o estándar. Se estimó el tiempo básico de cada tarea, a través de la sumatoria los tiempos básicos de cada uno de sus elementos. Posteriormente, con la aplicación de la ecuación (3), se asignaron los suplementos de tiempo por descanso (porcentajes) calculados previamente; obteniéndose así, los tiempos estándar del proceso y en consecuencia, el tiempo estándar del proceso en estudio.

Tiempo tipo=tiempo básico* $(1+$ suplemento de tiempo por descanso $)$

Ecuación 3. Cálculo de tiempo tipo. Fuente: OIT (2005).

Asimismo, se identificó que la tarea de Preparación del yogurt es el cuello de botella del sistema; de modo que, es quien determina el índice de procesamiento de la línea en estudio, mismo que se halló con la ecuación (4). 


$$
\text { Índice de procesamiento }=\frac{1}{\text { tiempo de ciclo }}
$$

Ecuación 4. Índice de procesamiento. Fuente: Chase (2009).

Finalmente, se revisó el indicador de eficiencia del proceso, el cual Chase (2005) define como "la proporción de la producción real de un proceso en relación con algún parámetro" (p. 169); sin embargo, el autor en cuestión aclara que esta medida puede expresarse también como la ganancia o pérdida de un proceso, si no se maneja una producción estándar. Es decir, la relación entre las entradas y salidas del mismo, como lo evidencia la ecuación (5).

$$
\% \text { Eficiencia }=\frac{\text { Salidas }}{\text { Entradas }} * 100
$$

Ecuación 5. Eficiencia. Fuente: Chase (2005).

Para lo anterior, se identificaron las cantidades o proporciones de materias primas que ingresan al sistema y las salidas de producto (yogurt), su densidad y con ello, su equivalente en masa, manejando como base un lote de procesamiento de 550L de leche; obteniéndose así, el valor en kilogramos (Kg.) de materias primas y producto terminado.

\section{RESULTADOS Y DISCUSIÓN}

Como se indicó, la empresa La Hacienda Productos Alimenticios se encuentra en proceso de ampliación de su portafolio de productos, por lo que se encuentra en proceso de adecuación de su infraestructura para incorporar nuevos procesos a su planta de producción; de modo que, en el momento, se ocupa de fabricar principalmente yogurt, en presentaciones de bolsas de $200 \mathrm{ml}$. El mercado que abastece la empresa en cuestión es netamente institucional; la planta de producción tiene un área total de 320,21 $\mathrm{m}^{2}$, distribuida en siete áreas: Almacén de materias primas, Laboratorio, Plataforma de recepción, Área de quesos, cuarto y tanques del yogurt, cuarto frío y zona de empaque. Para el desarrollo de su actividad productiva, la empresa cuenta con cuatro empleados de tiempo completo, uno de los cuales es profesional en Química de Alimentos, quien se encarga del trabajo de laboratorio y en general, de la supervisión del proceso. 


\section{Etapa 1. Información de las tareas.}

El proceso de fabricación del yogurt en la empresa La Hacienda consta de cuatro subprocesos: recepción de la leche cruda, alistamiento de materias primas, preparación del yogurt y finalmente, el empacado y almacenamiento.

- Recepción de la leche cruda: inicia con descarga de las cantinas de la leche en una plataforma de recepción de capacidad de 14 cantinas, que a su vez, tienen una de capacidad de 40L de leche cada una. Seguidamente, el profesional a cargo del laboratorio, toma dos muestras de leche de las cantinas, cada muestra contiene $20 \mathrm{ml}$ de leche cada cantina; seguidamente, lleva las muestras al laboratorio y realiza pruebas de tipo organolépticas y físico-químicas, tales como: nivel de acidez, pH, proporción de sólidos presentes y densidad. Una vez se aprueba la calidad de la leche, se descargan las cantinas en el tanque de recepción de la plataforma, que vía tubería (12m aprox.) envía su contenido al tanque 1 del yogurt.

- Alistamiento de materias primas: en esta operación, se determina en el almacén de materias primas, la cantidad de azúcar y estabilizante necesarias para el lote de leche en procesamiento. Luego, el operario encargado, lleva la materia prima correspondiente al cuarto del yogurt, donde calcula y pesa las cantidades de colorante, saborizante y cultivo de bacterias requeridas.

- Preparación del yogurt: en este subproceso, se lleva a cabo básicamente la transformación de la leche cruda en yogurt. Inicialmente, la leche contenida en el tanque 1 , se precalienta a una temperatura de $25^{\circ} \mathrm{C}$ mediante la inyección de vapor caliente a las paredes del tanque, luego se adicionan las cantidades de azúcar y estabilizante previamente llevadas al cuarto del yogurt.

- Luego del precalentamiento, la mezcla debe pasteurizarse, para ello, se incrementa nuevamente la temperatura de la mezcla hasta $\operatorname{los} 85^{\circ} \mathrm{C}$, en ese punto, la leche se deja alrededor de 15 minutos, para eliminar cualquier bacteria presente y además, generar la acidez necesaria. Posteriormente, se abre la válvula de agua fría del tanque, para enfriar sus paredes y lograr que la mezcla alcance los $60^{\circ} \mathrm{C}$. A esa temperatura, se incorpora el cultivo de bacterias, con el cual se transforma la mezcla en yogurt, y se deja incubar (proliferación de las bacterias) durante cuatro horas a una temperatura que puede oscilar entre los 40 y $45^{\circ} \mathrm{C}$, luego, la mezcla se enfría de forma natural (en la noche).

- Seguidamente, el operario encargado con ayuda de un agitador manual, rompe el coágulo en que se convierte la mezcla en la incubación, homogeniza el yogurt y con un acidómetro en el cuarto del yogurt, verifica que su nivel de acidez esté entre los $80^{\circ} \mathrm{Th}-90^{\circ} \mathrm{Th}$; de lo contrario, el lote en proceso no es conforme. De ser conforme, se adiciona el saborizante y el colorante al yogurt. 
- Empaque y almacenamiento: la operación de empaque comienza con la filtración del yogurt, que se da con su paso del tanque 1 al tanque 2, mediante un tubo de conexión que contiene dentro un filtro. El tanque 2 envía de forma intermitente el producto a la tolva de la máquina empacadora, la cual empaca el yogurt en bolsas plásticas de capacidad de $200 \mathrm{ml}$; cada que se empacan cinco bolsas, el operario pesa una bolsa de yogurt en una gramera, con el fin de verificar que su peso esté entre los 195 y $205 \mathrm{ml}$ (requisito de calidad).

- Otro operario, en la mesa de inspección de calidad, examina el estado de la bolsa de producto, ubicándolo bien sea en las canastas de producto conforme o en las de producto defectuoso. Finalmente, las canastas cuya capacidad es de 120 bolsas de yogurt (presentación de $200 \mathrm{ml}$ ), son llevadas al cuarto frío, donde se almacenan a una temperatura de $4^{\circ} \mathrm{C}$ hasta el momento en que se despachan al cliente.

La figura 2 presenta el diagrama de flujo del proceso en estudio, conformado por cuatro tareas y sus respectivos elementos, los cuales se resumen en cinco operaciones, cuatro inspecciones, una demora, tres transportes, dos almacenamientos cuatro tareas de tipo inspección - operación (ver tabla 3).

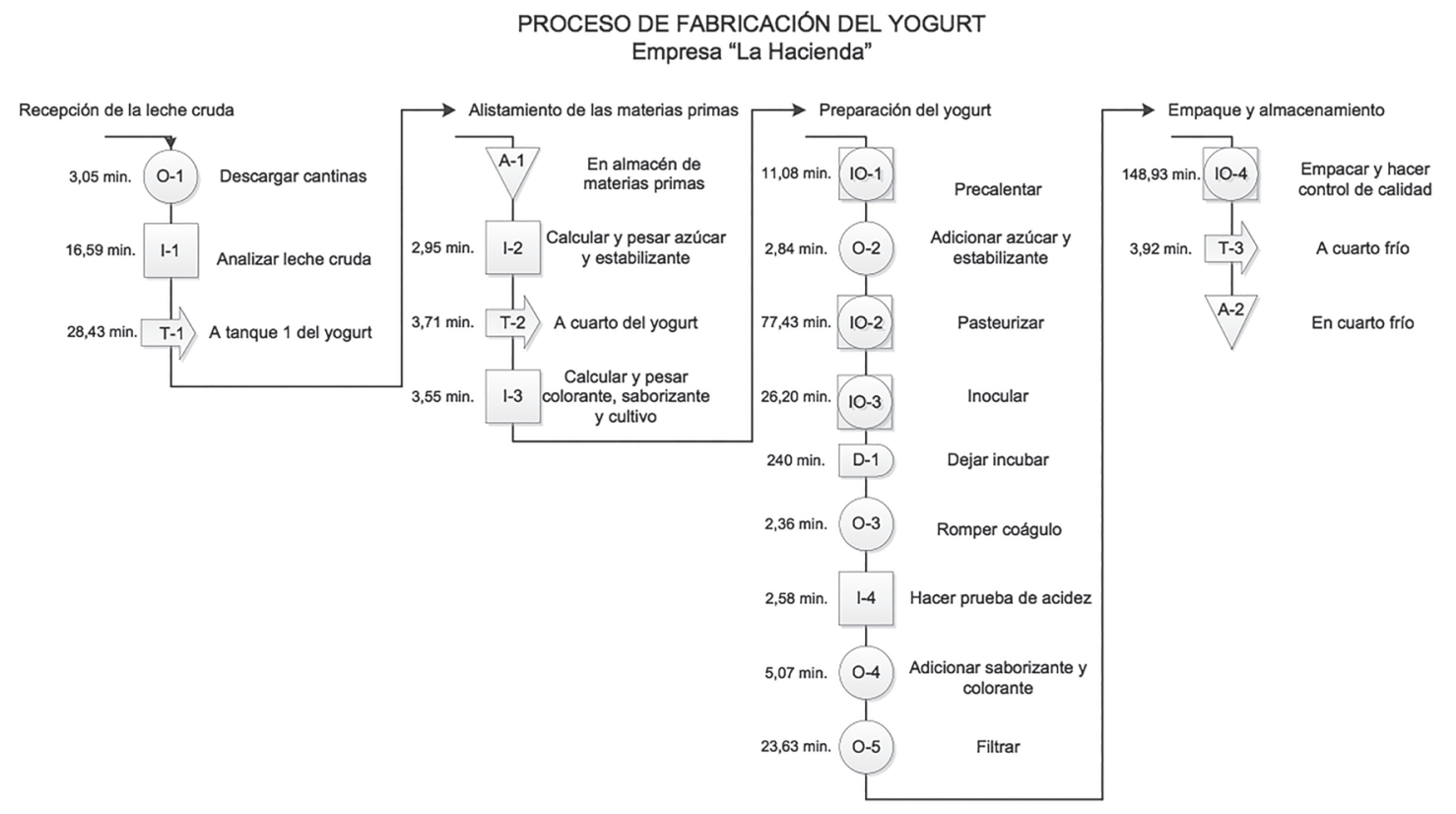

Figura 2. Diagrama de flujo del proceso. Fuente: elaboración propia. 


\begin{tabular}{|c|c|c|}
\hline \multicolumn{2}{|c|}{ RESUMEN } \\
\hline Evento & Símbolo & Cantidad \\
\hline Operación & & 5 \\
\hline Inspección & & \\
\hline Demora & & 4 \\
\hline Transporte & & 1 \\
\hline Almacenamiento & & 3 \\
\hline Total & & \\
\hline
\end{tabular}

Tabla 3. Resumen diagrama del proceso.

Fuente: elaboración propia.

Cabe destacar que los datos correspondientes a tiempo estándar presentados en el diagrama de flujo, se obtienen posteriormente y corresponden al tiempo estándar necesario para procesar un lote de $550 \mathrm{~L}$ de leche. La figura 3 presenta el diagrama de recorrido del proceso de fabricación del yogurt, a partir del cual se pueden observar las diferentes áreas de la planta de producción, a una escala de 2,3. 


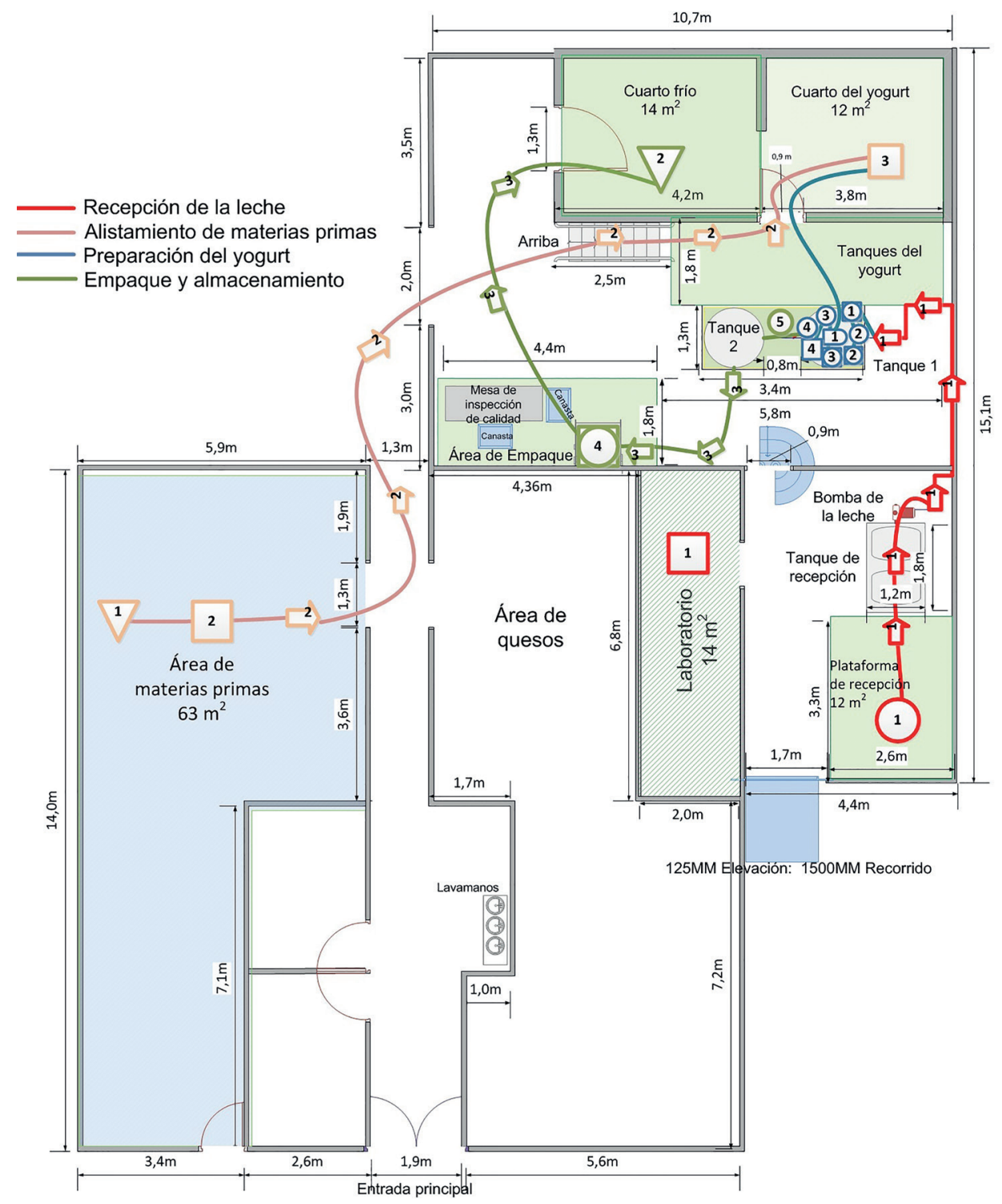

Figura 3. Diagrama de recorrido del proceso.

Etapa 2. Operaciones y elementos.

Fuente: elaboración propia.

Luego de conocer las etapas del proceso, se identificaron los elementos propios de cada una de las tareas, como se muestra en la figura 4. 


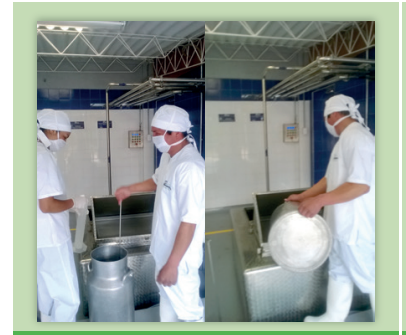

RECEPCIÓN DE LA LECHE CRUDA

- Descargar cantinas de la leche

- Analizar leche cruda

- Transportar leche a tanque 1 del yogurt

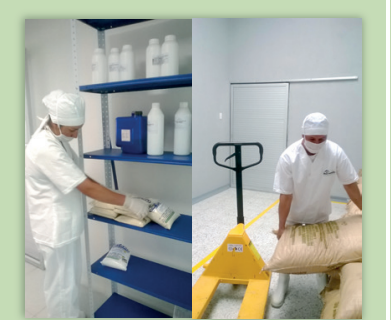

ALISTAMIENTO DE LAS MATERIAS PRIMAS

- Calcular y pesar azúcar y estabilizante

- Transportar materias primas a

cuarto del yogurt

- Calcular y pesar colorante,

saborizante y cultivo

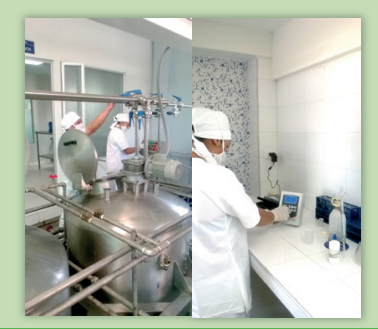

PREPARACIÓN DEL YOGURT

- Precalentar

- Adicionar azúcar y estabilizante

- Pasteurizar

- Inocular

- Dejar incubar

- Romper coágulo

- Hacer prueba de acidez

- Adicionar saborizante y

colorante

- Filtrar el yogurt

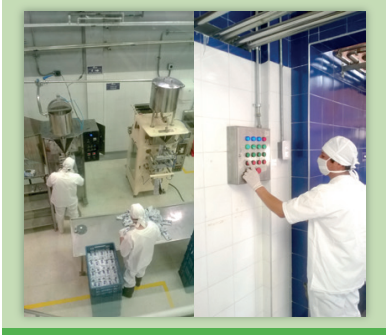

EMPAQUE Y ALMACENAMIENTO

- Empacar y hacer control de calidad

- Trasladar bolsas de yogurt a

cuarto frío

Figura 4. Elementos de la operación "Análisis de la leche cruda".

Fuente: elaboración propia.

\section{Etapa 3. Tamaño de la muestra}

Con la aplicación de la ecuación (1), se determinó que habrían de hacerse entre 8 y 17 observaciones de tiempo, como se muestra en la tabla 4.

\begin{tabular}{cc}
\hline \hline TAREA & TAMAÑO DE MUESTRA (N) \\
\hline \hline Recepción de la leche cruda & 16 \\
Alistamiento de las materias primas & 8 \\
Preparación del yogurt & 17 \\
Empaque y almacenamiento & 9 \\
\hline \hline
\end{tabular}

Tabla 4. Tamaños de muestra por operaciones del proceso.

\section{Etapa 4. Observaciones de tiempo}

Fuente: Elaboración propia.

Se realizaron los días miércoles y sábado desde las 7:30 am (hora en que se encendía el cronómetro) hasta cerca de las $2 \mathrm{pm}$, momento en que el proceso culminaba y se detenía el cronómetro, ya que

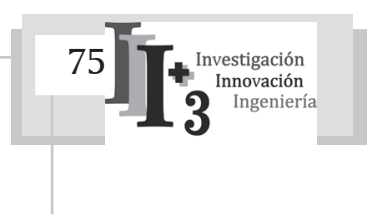


se procedía a dejar en incubación la mezcla. De esta manera, se obtuvo el registro de los tiempos observados promedio (ver tabla 5), de los elementos de cada tarea, para el procesamiento de un lote de fabricación de 550L de leche (máxima capacidad de la línea de producción).

\section{Etapa 5. Valoración del ritmo de trabajo}

Simultáneo a cada una de las tomas de tiempos, el investigador estimaba el factor de valoración que a su juicio, merecía el operario a cargo de la actividad en estudio, tomando en cuenta la escala de la Norma Británica, previamente mencionada; la tabla 5 presenta los factores de valoración promedio asignados a cada uno de los elementos correspondientes.

\section{Etapa 6. Tiempo básico o normal}

Con los factores de valoración y tiempos observados previamente estimados, se aplicó la ecuación (2) a cada uno de los elementos de las tareas, hallándose así, los tiempos básicos de dichos elementos de tarea, los cuales se presentan más adelante en la tabla 5.

\section{Etapa 7. Suplementos de tiempo}

Los suplementos de tiempo por descanso se aplicaron a cada uno de los elementos de las tareas, de acuerdo con las condiciones de trabajo identificadas en las etapas anteriores. Por lo que, se asignaron porcentajes de tiempo por descanso de entre el $11 \%$ y $62 \%$, como se muestra en la tabla 5 .

\section{Etapa 8. Tiempo tipo o estándar}

Finalmente, se asignaron los suplementos de tiempo por descanso a cada elemento de las operaciones del proceso, a través de la aplicación de la ecuación (3), obteniéndose así que el tiempo tipo o estándar del proceso del yogurt en la empresa La Hacienda para procesar un lote de 550L de leche es de 601,32 minutos, lo que equivale a 10 horas, 2 minutos y 19 segundos y a una producción de 3052 bolsas de yogurt de $200 \mathrm{ml}$ conformes. 


\begin{tabular}{|c|c|c|c|c|c|c|c|}
\hline TAREA & ELEMENTO & $\begin{array}{c}\text { TIEMPOS } \\
\text { OBSER- } \\
\text { VADOS (MIN/ } \\
\text { LOTE } 550 \mathrm{~L} \text { ) }\end{array}$ & $\begin{array}{l}\text { FACTOR DE } \\
\text { VALORACIÓN }\end{array}$ & $\begin{array}{l}\text { TIEMPOS } \\
\text { BÁSICOS } \\
\text { (MIN/LOTE } \\
550 \text { L) } \\
\end{array}$ & $\begin{array}{l}\text { SUPLEMENTO } \\
\text { DE TIEMPO } \\
\text { POR DESCANSO }\end{array}$ & $\begin{array}{c}\text { TIEMPO } \\
\text { ESTÁNDAR } \\
\text { (MIN/LOTE } \\
550 \mathrm{~L}) \\
\end{array}$ & $\begin{array}{c}\text { TIEMPO } \\
\text { ESTÁNDAR } \\
\text { (MIN/LOTE } \\
550 \mathrm{~L} \text { ) } \\
\end{array}$ \\
\hline \multirow{3}{*}{$\begin{array}{l}\text { Recepción de } \\
\text { la leche cruda }\end{array}$} & $\begin{array}{l}\text { Descargar canti- } \\
\text { nas de la leche }\end{array}$ & 2,376 & $98 \%$ & 2,328 & $31 \%$ & 3,05 & \multirow{3}{*}{48,07} \\
\hline & $\begin{array}{l}\text { Analizar leche } \\
\text { cruda }\end{array}$ & 19,490 & $76 \%$ & 14,813 & $12 \%$ & 16,59 & \\
\hline & $\begin{array}{c}\text { Transportar leche } \\
\text { a tanque } 1\end{array}$ & 28,498 & $86 \%$ & 24,509 & $16 \%$ & 28,43 & \\
\hline \multirow{4}{*}{$\begin{array}{l}\text { Alistamiento } \\
\text { de las mate- } \\
\text { rias primas }\end{array}$} & $\begin{array}{l}\text { Calcular y pesar } \\
\text { azúcar y estabili- } \\
\text { zante }\end{array}$ & 3,347 & $78 \%$ & 2,611 & $13 \%$ & 2,95 & \multirow{3}{*}{10,21} \\
\hline & $\begin{array}{l}\text { Transportar ma- } \\
\text { terias primas a } \\
\text { cuarto del yogurt }\end{array}$ & 2,436 & $94 \%$ & 2,290 & $62 \%$ & 3,71 & \\
\hline & $\begin{array}{l}\text { Calcular y pesar } \\
\text { colorante, sabori- } \\
\text { zante y cultivo }\end{array}$ & 3,561 & $89 \%$ & 3,170 & $12 \%$ & 3,55 & \\
\hline & Precalentar & 10,085 & $99 \%$ & 9,984 & $11 \%$ & 11,08 & \multirow{8}{*}{391,19} \\
\hline \multirow{7}{*}{$\begin{array}{l}\text { Preparación } \\
\text { del yogurt }\end{array}$} & $\begin{array}{l}\text { Adicionar azúcar } \\
\text { y estabilizante }\end{array}$ & 2,698 & $94 \%$ & 2,536 & $12 \%$ & 2,84 & \\
\hline & Pasteurizar & 93,424 & $74 \%$ & 69,134 & $12 \%$ & 77,43 & \\
\hline & Inocular & 22,982 & $100 \%$ & 22,982 & $14 \%$ & 26,2 & \\
\hline & Dejar incubar & \multicolumn{4}{|c|}{ OPERACIÓN DETERMINÍSTICA } & 240 & \\
\hline & Romper coágulo & 2,311 & $92 \%$ & 2,126 & $11 \%$ & 2,36 & \\
\hline & $\begin{array}{l}\text { Hacer prueba de } \\
\text { acidez }\end{array}$ & 2,451 & $94 \%$ & 2,304 & $12 \%$ & 2,58 & \\
\hline & $\begin{array}{l}\text { Adicionar sabori- } \\
\text { zante y colorante }\end{array}$ & 4,661 & $98 \%$ & 4,568 & $11 \%$ & 5,07 & \\
\hline \multirow{4}{*}{$\begin{array}{l}\text { Empaque } \\
\text { y almace- } \\
\text { namiento }\end{array}$} & Filtrar el yogurt & 19,896 & $107 \%$ & 21,288 & $11 \%$ & 23,63 & \multirow[b]{3}{*}{151,85} \\
\hline & $\begin{array}{l}\text { Empacar y hacer } \\
\text { control de calidad }\end{array}$ & 132,594 & $96 \%$ & 127,291 & $17 \%$ & 148,93 & \\
\hline & $\begin{array}{l}\text { Trasladar bolsas } \\
\text { de yogurt a cuar- } \\
\text { to frío }\end{array}$ & 3,108 & $81 \%$ & 2,517 & $16 \%$ & 2,92 & \\
\hline & \multicolumn{6}{|c|}{ TOTAL } & 601,32 \\
\hline
\end{tabular}

Tabla 5. Resumen estudio de tiempos.

Fuente: Elaboración propia. 
Como el tamaño de los lotes de producción que se procesan es variable (oscila entre 480L y 550L de leche) por la disponibilidad de la leche (principal materia prima del proceso) en la zona, se determinó que el tiempo estándar calculado, se manejaría sobre una base de procesamiento de $550 \mathrm{~L}$ de leche, que equivale a 3052 bolsas de yogurt de $200 \mathrm{ml}$.

Lo anterior, basados en que durante el período de tiempo en que se hicieron las observaciones de tiempo, en promedio el 5,06\% de la producción total fue producto no conforme, es decir, no cumplió con las especificaciones de calidad correspondientes.

\section{Índice de procesamiento e indicador de eficiencia}

De acuerdo con Chase (2005), el cuello de botella es el recurso que limita la capacidad del sistema y genera sobrecarga, por lo que es el que mayor tiempo consume. Luego, se puede inferir, que la tarea de Preparación del yogurt es el cuello de botella del sistema, ya que esta consume el 65\% del tiempo de procesamiento del proceso.

Tomando en cuenta que, el tiempo de ciclo de la tarea 'cuello de botella' es de 391,19 min., para procesar un lote de 550L de leche que equivale a una producción diaria promedio de 3052 bolsas de yogurt de $200 \mathrm{ml}$, se estimó con la aplicación de la ecuación (4) que el índice de procesamiento de la línea de producción del yogurt es de 468 bolsas de yogurt/hora.

$$
\text { Indice de procesamiento }=\frac{1}{\left(\frac{391,19 \text { min. }}{3052 \text { bolsas }}\right)}=7,8 \frac{\text { bolsas }}{\text { min. }}=468 \frac{\text { bolsas }}{\text { hora }}
$$

En cuanto al cálculo del indicador de eficiencia del proceso, se estableció gracias a información suministrada por la empresa, que un lote de $550 \mathrm{~L}$ de leche consume las cantidades o proporciones de materias primas que se presentan en la tabla 6 . 


\begin{tabular}{ccc}
\hline \hline & ENTRADAS & \\
\hline \hline Materia prima & Cantidad/550L leche & Total $(\mathrm{Kg} / 550 \mathrm{~L})$ \\
Azúcar & $12 \%$ & 75,665 \\
Estabilizante & $15 \mathrm{~g}$ & 0,206 \\
Cultivo de bacterias & $6 \mathrm{~g}$ & 0,007 \\
Saborizante & $15 \mathrm{~g}$ & 0,206 \\
Colorante & $8 \mathrm{~g}$ & 0,11 \\
Leche & $566.500 \mathrm{~g}$ & 566,5 \\
& & 642,694 \\
Yogurt & Total Entradas & 630,543 \\
& Tolidas & 630,543 \\
\hline
\end{tabular}

Tabla 6. Materias primas.

Fuente: Elaboración propia a partir de información suministrada por la empresa.

A partir de tal información, y de las densidades correspondientes de dichas materias primas, se estimó que para fabricar 630,543 $\mathrm{Kg}$ de yogurt (3052 bolsas de $200 \mathrm{ml}$ ), se deben ingresar al sistema $642,694 \mathrm{Kg}$. de materias primas; luego, se pudo establecer que la eficiencia del proceso de fabricación del yogurt en la empresa La Hacienda es del 98,1\%.

$$
\% \text { Eficiencia }=\frac{630,543 \mathrm{~kg} \text { Leche }}{642,694 \mathrm{~kg} \text { Materias Primas }} * 100=98,1 \%
$$

\section{CONCLUSIONES}

Con el desarrollo de la investigación, se pudo establecer que la capacidad real de producción de la línea del yogurt en la empresa es de 468 bolsas de yogurt de $200 \mathrm{ml} /$ hora; puesto que, el tiempo estándar para procesar un lote de 550L de leche cruda es de 601,32 min., lo que equivale a una producción de 3052 unidades de producto.

Igualmente, se determinó que el proceso del yogurt en la empresa La Hacienda, se compone de cuatro subprocesos: recepción de la leche cruda, alistamiento de materias primas, preparación del yogurt y por último, empaque y almacenamiento; cuyos elementos de tarea, corresponden a cinco (5) operaciones, cuatro (4) inspecciones, una (1) demora, tres (3) transportes y cuatro (4) actividades de tipo inspección-operación. 
Aún cuando la preparación del yogurt es el cuello de botella del sistema, es importante tener presente que uno de los elementos de esta tarea es la Incubación, que corresponde a una demora de tipo determinístico de 240 minutos, es decir, consume más del 60\% de la tarea; así, se recomienda investigar otros tipos de cultivo de bacterias o incluso, explorar otras condiciones de procesamiento que permitan efectuar la incubación en menor tiempo y sin afectar la calidad del producto.

Por otro lado, aunque el proceso opera con un grado de eficiencia del $98,1 \%$, lo que indica un bajo nivel de desperdicio en términos de consumo de materias primas, es importante destacar que además de las demoras generadas por la dificultad existente alrededor de la disponibilidad de la materia prima (leche) en el sector, se evidenciaron retrasos en la estación de Empaque y Almacenamiento, debido a las múltiples paradas de la máquina empacadora por desajuste en la medida de corte de las bolsas; de modo que, se recomienda a la empresa implementar herramientas como el TPM (Mantenimiento Productivo Total), para agilizar la operación en cuestión. TPM es una herramienta más de la gestión de procesos Lean Manufacturing.

Así mismo, el control de calidad que se efectúa es ineficaz, dado que además de que no es completo, se realiza a todas y cada una de las bolsas empacadas, consumiendo más tiempo de procesamiento. Finalmente, es necesario replantear la inspección del producto final, estimando un tamaño de muestra confiable que reduzca la cantidad de tiempo que en ello se invierte y desde luego, hacer más rigurosa la inspección integrando otros criterios como el corte de la bolsa, sus medidas, etc.

\section{REFERENCIAS BIBLIOGRÁFICAS}

Alonso, Á. (1998). Conceptos de organización industrial. Barcelona: Marcobo.

ANDI. (2014). Indicadores de coyuntura Colombia. Recuperado de http://www.andi.com.co/pages/proyectos_paginas/proyectos_detail.aspx?pro_id $=559 \& \mathrm{Id}=3 \&$ clase $=8 \&$ Tipo $=3$

ANDI. (2014). Informe Encuesta de Opinión Industrial Conjunta. Recuperado de http://www.andi.com.co/ pages/proyectos_paginas/proyectos_detail.aspx?pro_id $=559 \& \mathrm{Id}=3 \&$ clase $=8 \&$ Tipo $=3$

Chase, R. B., Aquilano, N. J. \& Jacobs, R. (2005). Administración de la producción y operaciones para una ventaja competitiva (Sexta ed.). Ciudad de México, México: McGraw Hill.

Departamento Administrativo Nacional de estadística (DANE). (2014). Encuesta Nacional Agropecuaria 2013. Recuperado de https://www.dane.gov.co/index.php/agropecuario/encuesta-nacional-agropecuaria 
Koonts, H., \& Weihrich, H. (1999). Administración, una perspectiva global (Sexta ed.). Ciudad de México, México: McGraw Hill.

Meyers, F. (2000). Estudio de tiempos y movimientos (Segunda ed.). México: Pearson Educación.

Ministerio de Comercio, Industria y Turismo. (2013). Informe de Industria. Recuperado de http://www. mincit.gov.co/publicaciones.php?id $=15695$

Montero, E. (Diciembre de 2013). Situación actual y perspectivas del sector lácteo a nivel mundial. En J.A. Madriz (Presidencia). Congreso Nacional Lechero. Conferencia llevada a cabo en el $19^{\circ}$ Congreso Nacional Lechero, San Jóse, Costa Rica. Recuperado de http://www.proleche.com/recursos/ documentos/congreso2013/Situacion_actual_y_perspectivas_del_sector_lacteo_a_nivel_mundial_Ing_Erick_Montero_Vargas_Costa_Rica.pdf

Mounthon Mejía, L. (21 de Marzo de 2014). Economía creció 4,3\% en 2013: Dane. El Heraldo. Recuperado de http://www.elheraldo.co/economia/economia-colombiana-crecio-43-en-2013-dane-146791

Niebel, B., \& Freivalds, A. (2004). Ingeniería industrial: métodos, estándares y diseño del trabajo (Onceava ed.). Ciudad de México, México: McGraw Hill.

OCDE/FAO. (2013). OCDE-FAO Perspectivas Agrícolas 2013-2022, Texcoco, Estado de México, Universidad Autónoma Chapingo. http://dx.doi.org/10.1787/agr_outlook-2013-es

Oficina Internacional del Trabajo. (2005). Introducción al estudio del trabajo (Cuarta ed.). México: Limusa.

Oficina Internacional del Trabajo. (1996). Introducción al estudio del trabajo (Cuarta ed.). Ginebra: Limusa.

Parada, J. (2014, 21 de marzo). Análisis: preocupa el balance negativo de la industria. El Tiempo. Recuperado de http://www.elheraldo.co/economia/economia-colombiana-crecio-43-en-2013-dane-146791

Sampieri, R., Collado, C., \& Baptista, P. (2010). Metodología de la investigación (Quinta ed.). México: Mc Graw-Hill. 